\title{
An Improved Eigenvalue Corrector Formula for Solving the Schrödinger Equation for Central Fields
}

\author{
By J. W. Cooley
}

1. Introduction. The wave equation for the nuclear motion of a diatomic molecule, in the Born-Oppenheimer approximation, is one which is encountered frequently in quantum-theoretical calculations. Numerical methods for its solution have been developed and used [1, 2, 3, 4] over many years for atomic problems where the potential is one obtained by Hartree-Fock self-consistent fields or the Thomas-Fermi-Dirac statistical field methods. Only relatively recently have computational techniques and the application of electronic computers enabled one to obtain accurate theoretical internuclear potentials at enough internuclear distances to calculate the wave functions for the motion of the nuclei and use them to obtain averages, over the nuclear motion, of molecular properties.

The present investigation is concerned with obtaining an accurate method for calculating the nuclear wave functions and vibrational-rotational energies of diatomic molecules with some economy in the number of values of the internuclear potential required. An improved formula for the correction of trial eigenvalues, which does not depend so much for its accuracy upon the smallness of the stepsize in the radial coordinate, and an analysis of the convergence of the procedure are given. A computer subroutine was written and numerical results obtained from it are described for a case where exact analytical solutions are known.

In what follows, the vibrational quantum number $v, v=0,1,2, \cdots$, will be used as a subscript to index the eigenvalues $E_{v}$ with the usual convention that $E_{0} \leqq E_{1} \leqq E_{2} \leqq \cdots$.

2. Method of Integration. The Schrödinger wave equation for the motion of the nuclei of a diatomic molecule, regarded as a symmetric top, can be expressed in polar spherical coordinates $R, \Theta, \Phi$ of one nucleus relative to the other and one additional coordinate $\phi$ giving the angular orientation of the electronic charge cloud about the internuclear axis. It has been shown [5] that the wave equation can be separated into its angular and radial parts and its solution may be expressed in the form

$$
\Psi=R^{-1} P(R) Y_{J \Lambda M}(\Theta, \Phi, \phi)
$$

where $P(R)$ is the solution of the one-dimensional radial Schroedinger equation

$$
\begin{aligned}
P^{(2)}(R) & =(U(R)-E) P(R), \\
P^{(n)} & =d^{n} P / d R^{n},
\end{aligned}
$$

the $Y_{J \Lambda M}$ 's are the hypergeometric functions, $\Lambda$ is the quantum number for the

Received May 15, 1961. The work presented in this paper was supported by the AEC Computing and Applied Mathematics Center, Institute of Mathematical Sciences, New York University, under contract with the U. S. Atomic Energy Commission. 
$z$-component of electronic angular momentum, and the radial internuclear potential, $U(R)$, is of the form

$$
U(R)=\left[J(J+1)-\Lambda^{2}\right] R^{-2}+Z_{a} Z_{b} R^{-1}+E_{\mathrm{el}}(R) .
$$

The second term is the electrostatic Coulomb repulsion energy of the nuclei and $E_{\text {el }}(R)$ is the electronic energy obtained by solving the electronic wave equation for each fixed internuclear distance $R$. The boundary conditions for (2.1) are $P(0)=0, P(R)$ bounded.

To get a difference equation whose solutions approximate the solutions of $(2.1)$ we let

$$
\begin{aligned}
& R_{i}=i h, \quad i=0,1,2, \cdots, n+1, \\
& P_{i}=P\left(R_{i}\right), \\
& U_{i}=U\left(R_{i}\right) .
\end{aligned}
$$

By dropping terms of the fourth order and higher in the series

$$
P_{i+1}+P_{i-1}=\sum_{k=0}^{\infty} \frac{2 h^{2 k}}{(2 k) !} P_{i}^{(2 k)}
$$

and by using the differential equation to replace $P_{i}{ }^{(2)}$, one obtains the simple integration formula

$$
P_{i+1}+P_{i-1}-2 P_{i}=h^{2}\left(U_{i}-E\right) P_{i}
$$

which has an error of approximately $\left(h^{4} / 12\right) P_{i}{ }^{(4)}$. A higher-order integration formula, which does not involve any more values of $P_{i}$, can be obtained by subtracting $h^{2} / 12$ times the series

$$
P_{i+1}^{(2)}+P_{i-1}^{(2)}=\sum_{k=0}^{\infty} \frac{2 h^{2 k}}{(2 k) !} P_{i}^{(2 k+2)}
$$

from (2.3). Then, dropping sixth and higher-order terms in $h$ gives

$$
Y_{i+1}+Y_{i-1}-2 Y_{i}=h^{2}\left(U_{i}-E\right) P_{i}
$$

where

$$
Y_{i}=P_{i}-\left(h^{2} / 12\right) P_{i}^{(2)}=\left[1-\left(h^{2} / 12\right)\left(U_{i}-E\right)\right] P_{i} .
$$

The error in (2.6) is approximately $-\left(h^{6} / 240\right) P_{i}{ }^{(6)}$. The integration formula (2.6), usually attributed to Numerov [6], involves the extra calculation of the $Y_{i}$ 's but reduces the number of points where values of $U(R)$ are required.

In the range $E>U(\infty)$, corresponding to unbound states of the two nuclei, solutions of (2.1) exist for all $E$ and can be approximated by simply using (2.4) or (2.6) to integrate outward, starting with the boundary values

$$
P_{0}=0, \quad P_{1}=\text { a small arbitrary number. }
$$

For $E<U(\infty)$, the two nuclei are bound together and solutions of (2.1) exist only for a set of discrete values of $E$, the eigenvalues of the problem. The method for calculating these eigenvalues and corresponding solutions, or eigenfunctions, is the subject of the present work. 
The boundary condition, $P(R)$ bounded, is approximated, as usual, by the conditions

$$
\begin{aligned}
P_{n+1} & =\text { a small arbitrary number } \\
P_{n} & =P_{n+1} \exp \left(R_{n+1} \sqrt{ } \overline{U_{n+1}-E}-R_{n} \sqrt{U_{n}-E}\right) .
\end{aligned}
$$

The second of these conditions results from the assumption that, at $R_{n}, U(R)$ is slowly approaching a constant.

The usual numerical procedure is to provide a first estimate of $E$ and integrate outward from $R=0$ to some point $R_{m}$, using starting values (2.8) and integration formula (2.4) or (2.6). Since (2.1) is homogeneous in $P(R)$, the resulting $P_{i}$ values may be replaced by $P_{i}{ }^{\text {out }}=P_{i} / P_{m}, i=1,2, \cdots, m$. With starting values (2.9), the same procedure is used to integrate inward from $R_{n+1}$ to $R_{m}$ and yields values $P_{m}^{i n}, i=n+1, n, \cdots, m$ such that $P_{m}^{i n}=P_{m}^{\text {out }}=1$. Then, a correction to $E$ is determined by the difference between the slopes of the two curves at the crossingpoint $R_{m}$ and the process is repeated until the two curves meet with the same derivative. The correction $D(E)$ [1] (See equation 6, p. 86) is usually calculated by using the formula

$$
D(E)=\left(P_{\text {out }}^{\prime}-P_{\text {in }}^{\prime}\right) / \int_{0}^{\infty}\{P(R)\}^{2} d R
$$

where the terms in the numerator are the derivatives at $R_{m}$ of the curves resulting from the outward and inward integration respectively.

Equation (2.10) has been derived from the differential equation and is claimed to give first-order convergence in the error. This means that if a trial solution satisfies the differential equation (2.1) at all points except $R_{m}$, then (2.10) deviates from the true correction by an amount which is proportional to the correction. In the next section, it will be shown that the eigenvalues of the difference equations, (2.4) or (2.6), are the zeros of appropriate functions $F(E)$ of the trial eigenvalue $E$ and that these zeros can conveniently be calculated by the Newton-Raphson method. The correction is

$$
D(E)=-F(E) / F^{\prime}(E)
$$

and, for $E$ near an eigenvalue $E_{0}$,

$$
D(E)=\Delta E+(\Delta E)^{2} Q+\text { high-order terms in } \Delta E
$$

where $\Delta E=E_{0}-E$ and

$$
Q=F^{\prime \prime}\left(E_{0}\right) / 2 F^{\prime}\left(E_{0}\right)
$$

Thus, the convergence of the present method is of second order in $\Delta E$. In Section 4 , an analysis of the behavior of $D(E)$ over the whole range of $E$ is given.

3. Correction formula. The integration formulas (2.4) and (2.6) can be written as systems of equations in the $P_{i}$ 's, and $Y_{i}$ 's respectively, as follows:

$$
\begin{aligned}
\left(2 h^{-2}+U_{1}-E\right) P_{1}-h^{-2} P_{2} & =0 \\
-h^{-2} P_{i-1}+\left(2 h^{-2}+U_{i}-E\right) P_{i}-h^{-2} P_{i+1} & =0 \\
-h^{-2} P_{n-1}+\left(h^{-2}+U_{n}-E\right) P_{n} & =0
\end{aligned}
$$


and

$$
\begin{array}{rlr}
\left\{2 h^{-2}+\left(U_{1}-E\right)\left[1-\left(h^{2} / 12\right)\left(U_{1}-E\right)\right]^{-1}\right\} Y_{1}-h^{-2} Y_{2} & =0 \\
-h^{-2} Y_{i-1}+\left\{2 h^{-2}+\left(U_{i}-E\right)\left[1-\left(h^{2} / 12\right)\left(U_{i}-E\right)\right]^{-1}\right\} Y_{i}-h^{-2} Y_{i+1} & =0 \\
-h^{-2} Y_{n-1}+\left\{h^{-2}+\left(U_{n}-E\right)\left[1-\left(h^{2} / 12\right)\left(U_{n}-E\right)\right]^{-1}\right\} Y_{n} & =0
\end{array}
$$

where $i=2,3, \cdots, n-1$ in both cases. A term involving $E$ has been omitted from the $n$th equation in both (3.1) and (3.2). It can easily be shown that if one has, as one should, started the inward integration at a large enough $R_{n}$ to justify the assumption used in forming the boundary condition (2.9), this omission is justified.

The procedure used here to derive and analyze the correction formula is an adaptation of a very general and effective technique, due to Löwdin [7], for calculating solutions of the Schroedinger equation. To apply Löwdin's method, consider the vector-matrix formulation of equations (3.1) or (3.2),

$$
\mathbf{M C}=\mathbf{0},
$$

where $\mathbf{0}$ is a null vector, $\mathbf{C}$ is a vector containing the $P_{i}$ 's or the $Y_{i}$ 's, as the case may be, and $\mathbf{M}$ is the symmetric matrix of coefficients which, for the present, may simply be regarded as functions of $E$. After an outward and an inward integration with a trial value of $E$, all equations except the $m$ th are satisfied. Now, assume that the first row of $\mathbf{M}$ and the first element of $\mathbf{C}$ correspond to the $m$ th equation and the $m$ th variable, respectively, in equations (3.1) or (3.2). Then, by partitioning the first index of $\mathbf{M}$ and $\mathbf{C}$, the result of the integration can be expressed,

$$
\left(\begin{array}{ll}
M_{11} & \mathbf{M}_{a 1}^{\dagger} \\
\mathbf{M}_{a 1} & \mathbf{M}_{a a}
\end{array}\right)\left(\begin{array}{c}
1 \\
\mathbf{C}_{a}
\end{array}\right)=\left(\begin{array}{c}
F(E) \\
0
\end{array}\right)
$$

where $F(E)$ is the amount by which the $m$ th equation of (3.1) or (3.2) is not satisfied when integrating with the trial value $E$. It is assumed that the first element of $\mathrm{C}$, which is $P_{m}$ or $Y_{m}$, is nonzero and, for convenience, it is set equal to 1 . Equation (3.4) may be written in the form

$$
\begin{aligned}
F(E) & =M_{11}+\mathbf{M}_{a 1}^{\dagger} \mathbf{C}_{a} \\
0 & =\mathbf{M}_{a 1}+\mathbf{M}_{a a} \mathbf{C}_{a} .
\end{aligned}
$$

The function $F(E)$, whose zeros are the eigenvalues of the difference equations, is defined by (3.5) in terms of $\mathbf{C}_{a}$ which, in turn, is defined as a solution of (3.6). Furthermore, if $E$ is such that $\left|\mathbf{M}_{a a}\right| \neq 0$, then the solution $\mathbf{C}_{a}$ of (3.6) is unique and $F(E)$ is uniquely defined. An expression for $F^{\prime}(E)$ is obtained by differentiating (3.5) and (3.6), with respect to $E$,

$$
\begin{aligned}
F^{\prime}(E) & =M_{11}^{\prime}+\mathbf{M}_{a 1}^{\prime \dagger} \mathbf{C}_{a}+\mathbf{M}_{a 1}^{\dagger} \mathbf{C}_{a}^{\prime} \\
\mathbf{0} & =\mathbf{M}_{a 1}^{\prime}+\mathbf{M}_{a a}^{\prime} \mathbf{C}_{a}+\mathbf{M}_{a a} \mathbf{C}_{a}^{\prime}
\end{aligned}
$$


Multiplying (3.8) on the left by $\mathbf{C}_{a}^{\dagger}$ gives

$$
\mathbf{0}=\mathbf{C}_{a}^{\dagger} \mathbf{M}_{a 1}^{\prime}+\mathbf{C}_{a}^{\dagger} \mathbf{M}_{a a}^{\prime} \mathbf{C}_{a}+\mathbf{C}_{a}^{\dagger} \mathbf{M}_{a a} \mathbf{C}_{a}{ }^{\prime}
$$

From (3.6) and the fact that $\mathbf{M}_{a a}$ is symmetric it is seen that if (3.7) and (3.9) are added, the terms containing $\mathbf{C}_{a}{ }^{\prime}$ cancel and the result is

$$
F^{\prime}(E)=M_{11}^{\prime}+\mathbf{M}_{a 1}^{\prime \dagger} \mathbf{C}_{a}+\mathbf{C}_{a}^{\dagger} \mathbf{M}_{a 1}^{\prime}+\mathbf{C}_{a}^{\dagger} \mathbf{M}_{a a}^{\prime} \mathbf{C}_{a}
$$

which can be written

$$
F^{\prime}(E)=\mathbf{C}^{\dagger} \mathbf{M}^{\prime} \mathbf{C} .
$$

In the two cases considered here, only diagonal elements of $\mathbf{M}$ depend upon $E$ so that the correction formula $(2.11)$ can be written

$$
D(E)=-F(E) / \sum_{i=1}^{n} C_{i}^{2} M_{\imath i}^{\prime}
$$

In the case of (3.1), the derivatives of the diagonal elements of $\mathbf{M}$ are all equal to -1 so (3.12) becomes

$$
D(E)=\left[\left(-P_{m-1}+2 P_{m}-P_{m+1}\right) h^{-2}+\left(U_{m}-E\right) P_{m}\right] / \sum_{i=1}^{n} P_{i}^{2} .
$$

It is of some interest to compare this with the correction formula (2.10). If, in (2.10), central differences at $R_{m}$ are used to estimate the derivatives and the trapezoidal rule is used to estimate the integral in the denominator, then (2.10) is the same as (3.13). Therefore, use of the integration formula (3.1) and correction formula (2.10) as described above yields a second-order process in $\Delta E$.

In the case of the Numerov integration formula, where equations (3.2) are solved, the derivatives of the diagonal elements of $\mathbf{M}$ are

$$
M_{i i}^{\prime}=-\left[1-\left(h^{2} / 12\right)\left(U_{i}-E\right)\right]^{-2}, \quad i=1,2, \cdots, n .
$$

By substituting (2.7) the correction formula (3.12) may be written

$$
D(E)=\left[\left(-Y_{m-1}+2 Y_{m}-Y_{m+1}\right) h^{-2}+\left(U_{m}-E\right) P_{m}\right] / \sum_{i=1}^{n} P_{i}{ }^{2} .
$$

Unlike the case with the simpler integration formula (3.1), this is not the correction one would obtain from (2.10) by using the most natural higher-order estimates of the quantities in (2.10).

4. Convergence. When the Numerov integration formula (3.2) is used, a rigorous treatment of the convergence of the method would be quite complicated. Furthermore, since the difference in the two methods considered is a term of order $h^{4}$ in the solution $P(R)$ and since both are second-order processes in $\Delta E$, one is justified in assuming that the essential features of the convergence of the two procedures will be about the same. Therefore, the convergence of the method using equations (3.1) will be considered here. 
To get expressions for $F(E), F^{\prime}(E)$ and $D(E)$, let

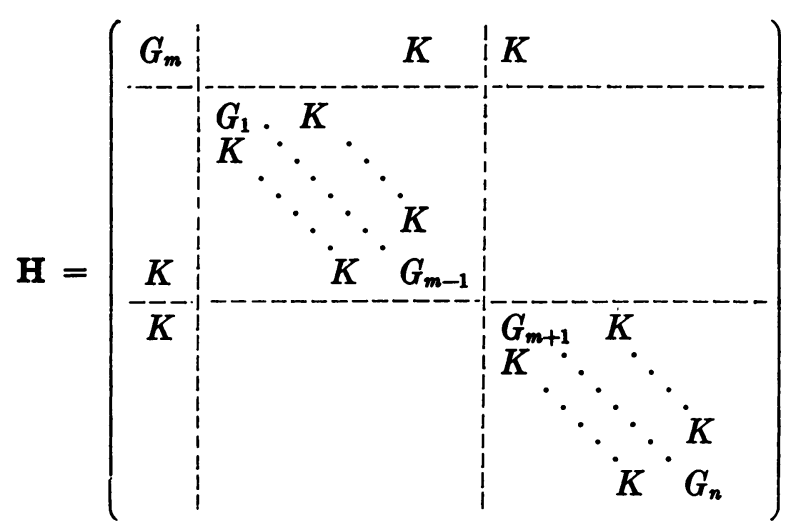

where

$$
\begin{aligned}
K & =-h^{-2} \\
G_{i} & =2 h^{-2}+U_{i}, \quad i=1,2, \cdots, n-1 \\
G_{n} & =h^{-2}+U_{n}
\end{aligned}
$$

and where the blank spaces denote zero elements. Then, for equations (3.1),

$$
\mathbf{M}=\mathbf{H}-\mathbf{I} \boldsymbol{E}
$$

where $I$ is the identity matrix. The symmetric matrix $\mathbf{M}_{a a}$ can be diagonalized by an orthonormal matrix $\mathbf{U}$, whose columns are eigenvectors of both $\mathbf{M}_{a a}$ and $\mathbf{H}_{a a}$. Thus,

$$
\mathbf{M}_{a a}=\mathbf{U} \mathbf{\Lambda} \mathbf{U}^{\dagger}
$$

where $\mathbf{\Lambda}$ is a diagonal matrix with the eigenvalues of $\mathbf{M}_{a a}$ on its diagonal. Then, if no eigenvalue of $\mathbf{M}_{a a}$ is zero, a solution of (3.6) exists and can be written

$$
\mathbf{C}_{a}=-\mathbf{M}_{a a}^{-1} \mathbf{M}_{a 1}=-\mathbf{U} \mathbf{\Lambda}^{-1} \mathbf{U}^{\dagger} \mathbf{M}_{a 1} \text {. }
$$

Letting

$$
\mathbf{V}=\mathbf{U}^{\dagger} \mathbf{M}_{a 1}
$$

and substituting (4.4) for $\mathrm{C}_{a}$ in (3.5), one obtains

$$
\begin{aligned}
F(E) & =M_{11}-\mathbf{V}^{\dagger} \mathbf{\Lambda}^{-1} \mathbf{V} \\
& =M_{11}-\sum_{v} V_{v}{ }^{2} \lambda_{v}^{-1} .
\end{aligned}
$$

As one can see from (4.2), the diagonal elements of $\mathbf{\Lambda}$ are

$$
\lambda_{v}=e_{v}-E
$$

where $e_{v}, v=1,2, \cdots, n-1, e_{v}<e_{v+1}$, are the eigenvalues of $\mathbf{H}_{a a}$, the matrix obtained by deleting the first row and column of $\mathbf{H}$. Hence,

$$
F(E)=2 h^{-2}+U_{m}-E-\sum_{v} V_{v}^{2}\left(e_{v}-E\right)^{-1}
$$


and

$$
F^{\prime}(E)=-1-\sum_{v} V_{v}^{2}\left(e_{v}-E\right)^{-2}
$$

The set of points $R_{1}, \cdots, R_{m-1}, R_{m+1}, \cdots, R_{n}$, referred to by the subscript " $a$ " in the vectors and matrices above, may be partitioned into the set of points $R_{1}, \cdots, R_{m-1}$, used in the outward integration, and the set of points $R_{m+1}, \cdots$, $R_{n}$, used in the inward integration. Letting "out" and "in" subscripts denote the sub-vectors and sub-matrices resulting from this partitioning, $\mathbf{H}_{a a}$ can be written

$$
\mathbf{H}_{a a}=\left(\begin{array}{cc}
\mathbf{H}_{\mathrm{out}} & \mathbf{0} \\
\mathbf{0} & \mathbf{H}_{\mathrm{in}}
\end{array}\right)
$$

where $\mathbf{H}_{\text {out }}$ and $\mathrm{H}_{\text {in }}$ are the block matrices lying on the diagonal in (4.1) and enclosed by the dashed lines. By partitioning in this manner, (4.3) can be written

$$
\begin{aligned}
& \mathbf{M}_{\text {out }}=\mathbf{U}_{\text {out }} \mathbf{\Lambda}_{\text {out }} \mathbf{U}_{\text {out }}^{\dagger} \\
& \mathbf{M}_{\text {in }}=\mathbf{U}_{\text {in }} \boldsymbol{\Lambda}_{\text {in }} \mathbf{U}_{\text {in }}^{\dagger}
\end{aligned}
$$

where the columns of $\mathbf{U}_{\text {out }}$ and $U_{\text {in }}$ are the eigenvectors of $\mathbf{H}_{\text {out }}$ and $\mathbf{H}_{\text {in }}$ respectively. Therefore, $e_{v}, v=1,2, \cdots, n-1$, are the eigenvalues of $\mathbf{H}_{\text {out }}$ and $\mathbf{H}_{\text {in }}$. From the form of $\mathbf{H}$, it is evident that the $e_{v}$ 's are the values of $E$ which, on the outward and inward integrations, respectively, lead to $P_{m}=0$, which is contrary to the assumption that the first element of $\mathbf{C}$ is nonzero.

To show that $F(E)$ is undefined at and only at the eigenvalues $e_{v}$ it is necessary to show that no $V_{v}$ is zero in (4.8). This is evident since (4.5) and the form of $\mathbf{M}_{a 1}$ imply that the elements of $\mathbf{V}$ are $-h^{-2}$ times the elements in the last row of $\mathbf{U}_{\text {out }}$ and the first row of $U_{\text {in }}$. If any such element of $U_{\text {out }}$ or $U_{\text {in }}$ were zero, then, since $\mathbf{M}_{\text {out }}$ and $\mathbf{M}_{\text {in }}$ are tridiagonal matrices, the column containing that element would be zero. This, of course, is not so, since these columns are the eigenvectors of $\mathbf{M}_{\text {out }}$ and $\mathbf{M}_{\text {in }}$.

It may be of some interest to show how the characteristic polynomial,

$$
P(E)=|\mathbf{M}| \text {, }
$$

of (3.1) is related to $F(E)$. From (4.3) and (4.5), one gets

$$
P(E)=\left|\begin{array}{cc}
M_{11} & \mathbf{V}^{\dagger} \\
\mathbf{V} & \mathbf{\Lambda}
\end{array}\right|
$$

which can be expanded in the elements of the first row and column of the determinant to give

$$
\begin{aligned}
P(\dot{E}) & =M_{11} \prod_{v^{\prime}} \lambda_{v^{\prime}}-\sum_{v} V_{v}^{2} \prod_{v^{\prime} \neq v} \lambda_{v^{\prime}} \\
& =\left(M_{11}-\sum_{v} V_{v}^{2} \lambda_{v}^{-1}\right) \prod_{v} \lambda_{v} .
\end{aligned}
$$

From (4.6) and (4.7), it is seen that

$$
P(E)=F(E) \cdot \prod_{v}\left(e_{v}-E\right) .
$$


Equations (4.8) and (4.9) enable one to determine the general behavior of $F(E)$, and $D(E)$. They show, first of all, that $F(E), F^{\prime}(E)$ and $D(E)$ are defined and continuous for all $E$ except $E=e_{v}, v=1,2, \cdots, n-1$ and that

$$
\begin{array}{ll}
F(E) \approx 2 h^{-2}+U_{m}-E & \text { for }|E| \text { large } \\
F(E)<2 h^{-2}+U_{m}-E & \text { for } E<e_{1} \\
F(E)>2 h^{-2}+U_{m}-E & \text { for } E>e_{n-1} \\
F(E) \approx-V_{v}^{2}\left(e_{v}-E\right)^{-1} & \text { for } E \approx e_{v} \\
F^{\prime}(E)<-1 & \text { for all } E \\
F^{\prime}(E) \approx-1 & \text { for }|E| \text { large } \\
F^{\prime}(E) \approx-V_{v}^{2}\left(e_{v}-E\right)^{-2} & \text { for } E \approx e_{v} .
\end{array}
$$

Therefore, in each of the intervals into which the points $e_{v}$ divide the $E$-axis, $F(E)$ is a continuous decreasing function which goes from positive to negative values. Hence, in each such interval, $F(E)$ must have one and only one zero. Let these zeros be denoted by $E_{v}, v=0,1,2, \cdots, n-1$ with $E_{0}<e_{1}, e_{v}<E_{v}<e_{v+1}$, $e_{n-1}<E_{n-1}$. The index $v$ is, therefore, the vibrational quantum number. From (4.16), it is apparent that the correction formula (3.13) has the following properties:

$$
\begin{array}{ll}
D(E) \approx E-e_{v} & \text { for } E \approx e_{v} \\
D(E) \approx 2 h^{-2}+U_{m}-E & \text { for }|E| \text { large } \\
D(E)<2 h^{-2}+U_{m}-E & \text { for } E<e_{1} \\
D(E)>2 h^{-2}+U_{m}-E & \text { for } E>e_{n-1} \\
D(E) \approx E_{v}-E & \text { for } E \approx E_{v} .
\end{array}
$$

The last condition is a general property of the Newton Raphson method (see (2.12)). A plot of $F(E)$ and $D(E)$, derived from the above analysis, may be expected to appear as shown in Figure 1.

A serious convergence difficulty is immediately obvious from the first property of $D(E)$. This indicates not only that $E \approx e_{v}$ leads to a gross underestimate of the correction for such values of $E$, but that the smallness of $D(E)$ cannot, by itself, be used as a convergence criterion. The condition $E \approx e_{v}$ can easily be detected by the existence of a large $F^{\prime}(E)$ and an increase in $D(E)$ from one iteration to the next. Another difficulty which may occur is a jumping from one branch of the $F(E)$ curve to another on successive iterations. This can cause one to miss some desired eigenvalues and waste computing effort in converging to eigenvalues which are not wanted or which have already been computed. Convergence problems, therefore, are related to the distribution of the vertical asymptotes at $e_{v}$, which are determined by the crossing point $R_{m}$, and to the selection of initial trial values of $E$.

To investigate the role of $R_{m}$, consider the convergence factor (2.13)

$$
Q=\frac{F^{\prime \prime}\left(E_{v}\right)}{2 F^{\prime}\left(E_{v}\right)}
$$




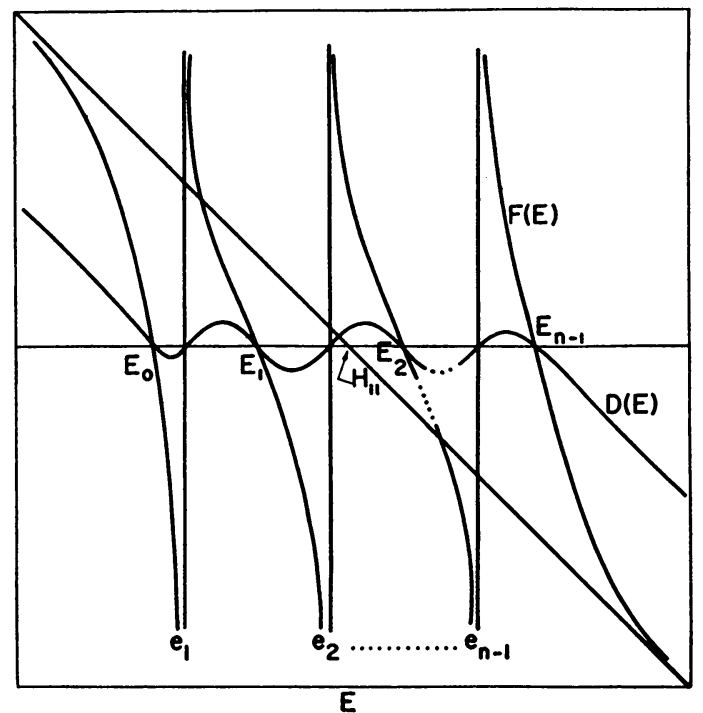

Frg. 1.-Behavior of $F(E)$ and $D(E)$ curves.

of the Newton-Raphson method. For $E_{v} \approx e_{v^{\prime}}$,

$$
Q \approx\left(e_{v^{\prime}}-E_{v}\right)^{-1}
$$

showing that $Q$ is large and convergence is poor if an eigenvalue $E_{v}$ is near a vertical asymptote. It was pointed out above that $e_{v}$ is a value of $E$ for which $P_{m}=0$ on the inward or outward integration. Therefore, $E_{v} \approx e_{v^{\prime}}$ is the situation where, for the correct solution, $P_{m} \approx 0$. In other words, convergence is poor if $R_{m}$ is near a node of the desired solution. Some practical means for selecting the crossing-point $R_{m}$, and initial estimates of $E$ are given in the next section.

5. Application. A computer subroutine was written which, when given a numerical potential, an initial estimate of $E$, and an $\epsilon$, integrates by the Numerov method (equation (2.6)) and uses (3.15) to correct $R$. After $D(E)$ starts decreasing from one iteration to the next, the convergence criterion $D(E) \leqq \epsilon$ is applied.

In order to keep $R_{m}$ as far as possible from a node of the solution and to acquire maximum significance where the solution is large, $R_{m}$ is selected during the integration as the point where $P_{i}$ is largest. Since, for the applications considered, this occurs at the outermost maximum point of $P_{i}$, the inward integration is performed first and $R_{m}$ is taken as the point at which $P_{i}$ stops increasing with decreasing $i$. The description of the behavior of $F(E)$, given in the previous section, is somewhat invalidated since, there, it was assumed that $R_{m}$ was held fixed while varying $E$. Instead, the $F(E)$ given by the present program will behave like $F(E)$ of the previous section in each $E$-interval where the variation of $E$ does not change $R_{m}$.

The data used here to demonstrate the method was obtained for the case where $U(R)$ of equation (2.1) is a Morse potential* [8]

* The parameters used are: $a=.711248, R_{e}=1.9975, D=188.4355$. These were obtained by fitting the Morse potential to a computed potential energy curve for $\mathrm{H}_{2}{ }^{+}$. Energies are given in units of $2 \mu$ atomic units, where $\mu$ is the reduced mass of the nuclei. 


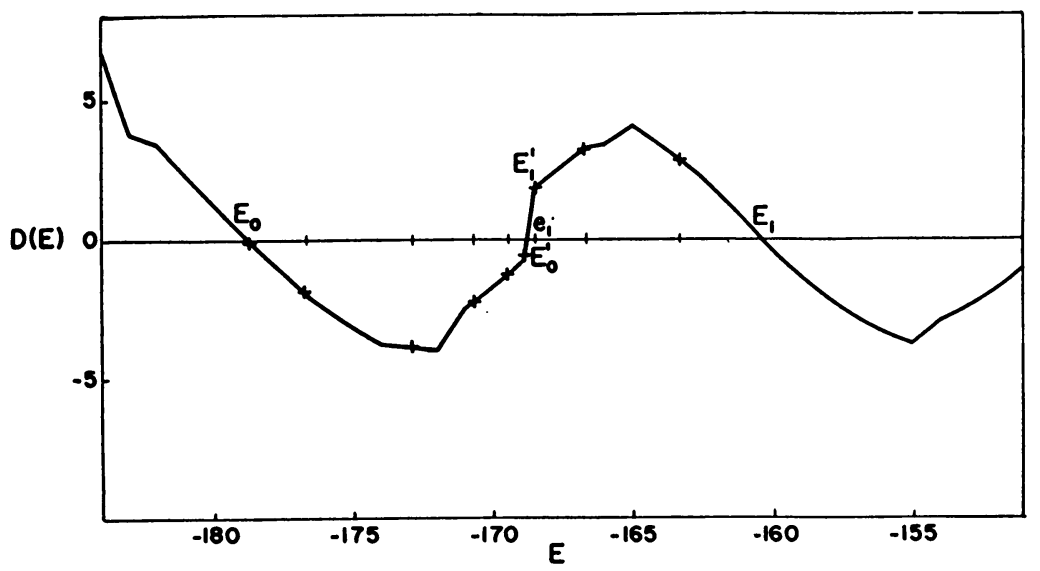

Fig. 2.-Graph of calculated $D(E)$. Crosses on curve denote successive iterates obtained with poor starting values $E_{0}^{\prime}$ and $E_{1}^{\prime}$.

TABLE 1

Successive Iterates Obtained with Poor First Estimates of $E_{0}$ and $E_{1}$

\begin{tabular}{|c|c|c|c|c|}
\hline$i$ & $E_{0}\left(i^{(i)}\right.$ & $F\left(E_{0}(i)\right)$ & $E_{1}\left(^{(i)}\right.$ & $F\left(E_{1}(i)\right)$ \\
\hline $\begin{array}{l}1 \\
2 \\
3 \\
4 \\
5 \\
6 \\
7 \\
7 \\
8 \\
9\end{array}$ & 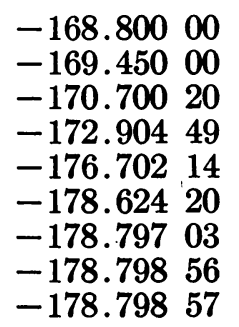 & $\begin{array}{l}-1470 \\
-727.9 \\
-350.7 \\
-113.0 \\
-26.24 \\
-2.042 \\
-.1786 \times 10^{-1} \\
-.5185 \times 10^{-4} \\
-.1228 \times 10^{-5}\end{array}$ & 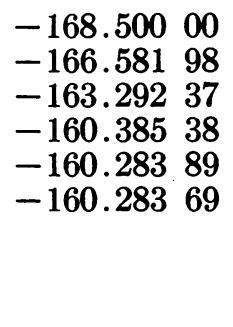 & $\begin{array}{l}456.7 \\
220.5 \\
58.21 \\
1.654 \\
\quad .0003 \\
\quad .1550 \times 10^{-4}\end{array}$ \\
\hline
\end{tabular}

TABLE 2

Dependence of Eigenvalues on $n$, the Number of Integration Points

\begin{tabular}{c|c|c|c|c|c}
\hline$n$ & $E_{0}$ & $E_{1}$ & $E_{2}$ & $E_{3}$ & $E_{4}$ \\
\hline 50 & -178.81052 & -160.35850 & -143.02059 & -126.83300 & -111.81094 \\
100 & -178.79924 & -160.28784 & -142.79397 & -126.31918 & -110.86348 \\
150 & -178.79866 & -160.28428 & -142.78276 & -126.29441 & -110.81921 \\
200 & -178.79857 & -160.28368 & -142.78090 & -126.29031 & -110.81191 \\
Exact & -178.79850 & -160.28182 & -142.77990 & -126.28824 & -110.80832 \\
\hline
\end{tabular}

$$
U(R)=D\left[1-\exp \left(-a\left(R-R_{e}\right)\right)\right]^{2}-D .
$$

Values of the analytic solution are given for comparison. The range $0<R \leqq 10$ was used in all cases.

A graph of the calculated $D(E)$ is given in Figure 2 for $E$ in the region of the two lowest eigenvalues. The effect of the shifting $R_{m}$ is evident. As one might expect, the discontinuities are large in the region of the $e_{v}$ 's, where the two solution curves 
cross with large slopes. On the other hand, there is a large region about each eigenvalue $E_{v}$ where such discontinuities are negligible and where $D(E)$ is almost a straight line of slope -1 and is, therefore, a good estimate of the necessary correction.

To show how the procedure converges when a poor first estimate of $E$ is used, the program was given a value of $E$ near $e_{1}$. As one can see in Table 1 where the successive trial values are given, and in Figure 2, $D(E)$ increases for a number of iterations and then goes down rapidly. Table 2 gives some of the eigenvalues obtained for $n=50,100,150$, and 200 points. Exact values of the analytic solution are given for comparison. Table 3 contains some of the values of the $v=0$ eigenfunctions which were obtained with 50,100 , and 200 points. Values obtained from the exact analytic solution are given in the last column. Figure 3 contains a graph of the potential (5.1) and the wave functions obtained for the $v=0,1$ and 2 states.

6. Conclusions. The above results indicate that fairly good accuracy can be achieved rapidly by the above procedure, but that good first trial values are quite important, nct only in reducing the number of iterations, but in assuring that no

TABLE 3

Dependence of Wave Function $P(R)$, for $v=0$, on $n$, the Number of Integration Points

\begin{tabular}{c|c|c|c|c}
\hline \multirow{2}{*}{$R$} & \multicolumn{5}{|c}{$P(R)$} \\
\cline { 2 - 4 } & \multicolumn{1}{|c|}{$n=50$} & \multicolumn{1}{|c}{$n=100$} & \multicolumn{1}{c}{$n=200$} & \multicolumn{1}{c}{ Exact } \\
\hline 1.2 & .0225903 & .0223875 & .0223754 & .0223746 \\
1.6 & .4840974 & .4858844 & .4859864 & .4859927 \\
2.0 & 1.3122858 & 1.3102630 & 1.3101471 & 1.3101405 \\
2.4 & .7329700 & .7347376 & .7348413 & .7348476 \\
2.8 & .1263448 & .1264907 & .1264998 & .1265004 \\
3.2 & .0089574 & .0089544 & .0089541 & .0089541 \\
\hline
\end{tabular}

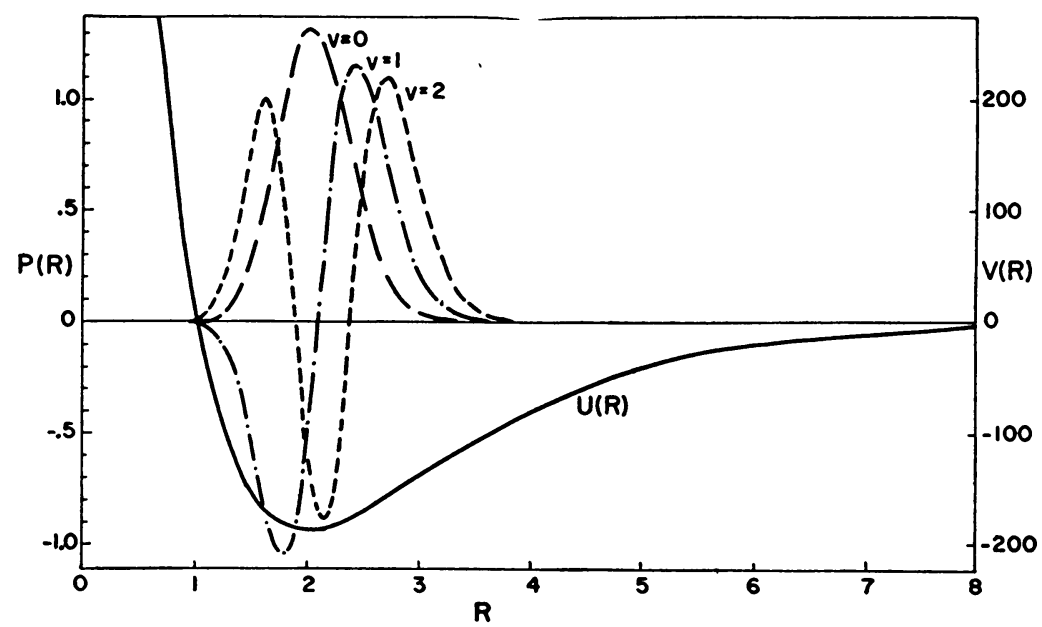

Fig. 3.-Plot of potential $U(R)$ and $v=0,1,2$ solutions. 
desired solutions are missed. Good first estimates of $E_{v}$ can be obtained by fitting an analytic potential for which eigenvalues are known and using it to obtain first estimates of the lowest eigenvalues. An extrapolation of calculated eigenvalues can then give an approximation to each new eigenvalue.

If it is inconvenient to obtain first estimates of $E$ in this manner, one can have the computer subroutine perform just one iteration for each of a series of values of $E$ to get a $D(E)$ curve. Then, in each interval where $D(E)$ changes from positive to negative, there is an eigenvalue for which a first estimate can be obtained by interpolation of $D(E)$. A count of the nodes in the solution, given by the subroutine, is a check on the vibrational quantum number assigned and assures that no solution is missed.

Institute of Mathematical Sciences

New York University

New York

1. D. R. Hartree, The Calculation of Atomic Structure, John Wiley and Sons, New York. 1957, p. 86.

2. E. C. Ridlex, "The self-consistent field for $\mathrm{Mo}^{+}$," Proc. Cambridge Philos. Soc. v. 51, 1955, p. 702 .

3. R. A. Rubenstein, M. Huse, \& S. Machlup, "Numerical solution of the Schroedinger equation for central fields," $M T A C$, v. $10,1956, \mathrm{p} .30$.

4. S. SkILlmaN, "Efficient method for solving atomic Schrödinger's equation," MTAC, v. 13, 1959, p. 299.

5. R. DE L. KRONIG \& I. I. RABI, "The symmetrical top in the undulatory mechanics," Phys. Rev., v. 29, 1927, p. 262.

6. B. NuMERov, Publs. observatoire central astrophys. Russ., v. 2, 1933, p. 188.

7. P. O. LöwdIN, An Elementary Iteration-Variation Procedure for Solving the Schroedinger Equation, Technical Note No. 11, Quantum Chemistry Group, Uppsala University, Uppsala, Sweden, 1958.

8. P. M. Morse, "Diatomic molecules according to the wave mechanics II. Vibrational levels," Phys. Rev., v. 34, 1929, p. 57. 